\title{
Minimal and Maximal Beta Open Sets
}

\author{
Qays Rashid Shakir \\ Operations Management Techniques Department-Technical College of Management Baghdad, \\ Baghdad-Iraq. \\ E-mail: qays.shakir@gmail.com.
}

\begin{abstract} properties of the concepts which presented in this paper. $\beta$-continuous

\section{Introduction}

Minimal and maximal sets play an important role in the researches of generalized topological spaces, Nakaoka and Oda introduced these concepts in [1] and [2] and they used them to investigate many topological properties. In this paper we introduced the notion of minimal $\boldsymbol{\beta}$-open and maximal $\boldsymbol{\beta}$-open and their complements.
\end{abstract}

The present paper deals and discusses new types of sets all of these concepts completely depended on the concept of Beta open set. The importance concepts which introduced in this paper are minimal $\boldsymbol{\beta}$-open and maximal $\boldsymbol{\beta}$-open sets. Besides, new types of topological spaces introduced

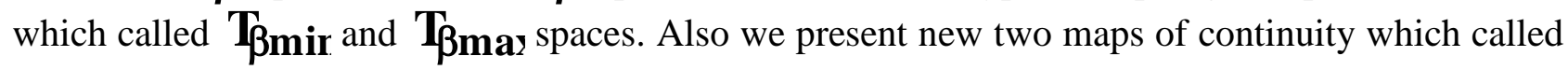
minimal $\boldsymbol{\beta}$-continuous and maximal $\boldsymbol{\beta}$-continuous. Additionally we investigated some fundamental

Keywords: minimal $\beta$-open set, maximal $\beta$-open set, minimal $\beta$-continuous and maximal

\section{Definition (1) [1]:}

A proper nonempty open subset $\mathrm{O}$ of a topological space $\mathrm{X}$ is said to be minimal open set if any open set which is contained in $\mathrm{O}$ is $\phi$ or $\mathrm{O}$.

\section{Definition (2) [2]:}

A proper nonempty open subset $\mathrm{O}$ of a topological space $\mathrm{X}$ is said to be maximal open set if any open set which is contains $\mathrm{O}$ is $\mathrm{O}$ or $\mathrm{X}$.

\section{Definition (3) [3]:}

A proper nonempty closed subset $\mathrm{O}$ of a topological space $\mathrm{X}$ is said to be minimal closed set if any closed set which is contained in $\mathrm{O}$ is $\phi$ or $\mathrm{O}$.

\section{Definition (4) [3]:}

A proper nonempty closed subset $\mathrm{O}$ of a topological space $\mathrm{X}$ is said to be maximal closed set if any closed set which is contains $\mathrm{O}$ is $\mathrm{O}$ or $\mathrm{X}$.

\section{Definition (5) [4]:}

Let A be a subset of a topological space X, then the union of all open subset of $X$ which contained in $\mathrm{A}$ is called the interior of $\mathrm{A}$ and denoted by $\mathbf{A}^{\mathbf{O}}$ and the intersection of all closed subset of $\mathrm{X}$ which contain $\mathrm{A}$ is called the closure of $\mathrm{A}$ and denoted by $\overline{\mathbf{A}}$.

Definition (6) [5]:

A subset $A$ of a space $X$ is called a $\boldsymbol{\beta}$-open set if $\mathbf{A} \subseteq \overline{\mathbf{A}}^{\mathbf{0}}$.The complement of a $\boldsymbol{\beta}$-open set is defined to be a $\boldsymbol{\beta}$-closed set.

Definition (7) [5]: Let $X$ and $Y$ be topological spaces and $f: X \rightarrow Y$ is a map then $f$ is called a $\boldsymbol{\beta}$-continuous function if $\mathbf{f}^{-1}(\mathrm{~A})$ is a $\boldsymbol{\beta}$-open set in $\mathrm{X}$ for every open set $\mathrm{A}$ in $\mathrm{Y}$.

Minimal and Maximal $\boldsymbol{\beta}$-open sets

\section{Definition (8):}

A proper $\boldsymbol{\beta}$-open subset $B$ of a topological space $X$ is said to be a minimal $\boldsymbol{\beta}$-open set if any $\boldsymbol{\beta}$-open set which is contained in $\mathrm{B}$ is $\boldsymbol{\phi}$ or $\mathrm{B}$.

\section{Definition (9):}

A proper nonempty $\boldsymbol{\beta}$-open subset $\mathrm{B}$ of a topological space $X$ is said to be a maximal $\boldsymbol{\beta}$-open set if any $\boldsymbol{\beta}$-open set which contains $\mathrm{B}$ is $\mathrm{X}$ or $\mathrm{B}$.

\section{Definition (10):}

A proper nonempty $\boldsymbol{\beta}$-closed subset $\mathrm{F}$ of a topological space $X$ is said to be a minimal $\boldsymbol{\beta}$-closed set if any $\boldsymbol{\beta}$-closed set which is contained in $\mathrm{F}$ is $\phi$ or $\mathrm{F}$. 


\section{Definition (11):}

A proper nonempty $\boldsymbol{\beta}$-closed subset $\mathrm{F}$ of a topological space $X$ is said to be a maximal $\boldsymbol{\beta}$-closed set if any $\boldsymbol{\beta}$-closed set which contains $\mathrm{F}$ is $\mathrm{X}$ or $\mathrm{F}$.

\section{Remarks (12):}

(1) The family of all minimal $\boldsymbol{\beta}$-open (resp. minimal $\boldsymbol{\beta}$-closed) sets of a topological space $X$ is denoted by $\mathbf{M} \beta \mathbf{O}(\mathbf{X})$ (resp. $\mathbf{M} \beta C(X)$ ).

(2) The family of all maximal $\boldsymbol{\beta}$-open (resp. maximal $\boldsymbol{\beta}$-closed) sets of a topological space $X$ is denoted by $\mathbf{M} \mathbf{Q} \mathbf{O X}$ )(resp. $\mathrm{M} \beta C(\mathrm{X})$ ).

\section{Remark (13):}

The concept of minimal $\boldsymbol{\beta}$-open, maximal $\boldsymbol{\beta}$-open, minimal $\boldsymbol{\beta}$-closed and maximal f-closed are independent of each other as in the following example. Example (14): let $X=\{a$, $\mathrm{b}, \mathrm{c}\}$ and

\section{$\tau=\{\phi, \quad\{a\}, \quad\{a, b\}, \quad X\}$ so $\beta O(X \neq\{\phi,\{a\}, a b\},\{a c\}, X\}$, \\ $\mathbf{M} \beta O(X)=\{\{\mathfrak{a}\}, \quad \mathbf{M} \beta \mathbf{C O X})=\{\{c\},\{b\}\}$ $M \beta O(X)=\{a, b\},\{a, c\}\}$, \\ $M \beta C(X)=\{b, c\}\}$}

So the following table show that the new sets are independent each other.

Table (1).

\begin{tabular}{|c|c|c|c|c|}
\hline & $\{a\}$ & $\{\mathrm{b}\}$ & $\{a, b\}$ & $\{\mathrm{b}, \mathrm{c}\}$ \\
\hline Minimal $\beta$-open & Yes & $\mathrm{N}_{0}$ & $\mathrm{~N}_{0}$ & $\mathrm{~N}_{0}$ \\
\hline Minimal B-closed & $\mathrm{N}_{0}$ & Yes & $\mathrm{N}_{0}$ & $\mathrm{~N}_{0}$ \\
\hline Maximal $\beta$-open & $\mathrm{N}_{0}$ & $\mathrm{~N}_{0}$ & Yes & $\mathrm{N}_{0}$ \\
\hline Maximal B-closed & $\mathrm{N}_{0}$ & $\mathrm{~N}_{0}$ & $\mathrm{~N}_{0}$ & Yes \\
\hline
\end{tabular}

\section{Theorem (15):}

Let $F$ be a subset of a topological space $X$, then $\mathrm{F}$ is a minimal $\boldsymbol{\beta}$-closed if and only if $\mathrm{X}$ $\mathrm{F}$ is maximal $\boldsymbol{\beta}$-open set.

\section{Proof:}

$\Longrightarrow$ let $\mathrm{F}$ be a minimal $\boldsymbol{\beta}$-closed, so $\mathrm{X}-\mathrm{F}$ is $\boldsymbol{\beta}$-open. We have to show that $\mathrm{X}-\mathrm{F}$ is maximal $\boldsymbol{\beta}$-open suppose not, so there is a $\boldsymbol{\beta}$-open subset $D$ of $X$ such that $\mathbf{X}-\mathbf{F} \subset \mathbf{D}$ hence
$\mathbf{X}-\mathbf{D} \subset \mathbf{F}$ and this contradict being $\mathrm{F}$ is minimal $\boldsymbol{\beta}$-closed.

$\Leftarrow$ let $\mathrm{F}$ be a $\boldsymbol{\beta}$-closed subset of $\mathrm{X}$, suppose that there is a $\boldsymbol{\beta}$-closed $\mathbf{K} \neq \boldsymbol{\phi}$ such that $\mathbf{K} \subset \mathbf{F}$ thus $\mathbf{X}-\mathbf{F} \subset \mathbf{X}-\mathbf{K}$ but $\mathrm{X}-\mathrm{K}$ is proper $\boldsymbol{\beta}$-open set. Contradiction to the assumption of being $\mathrm{X}-\mathrm{F}$ is maximal $\boldsymbol{\beta}$-open.

\section{Theorem (16):}

Let $\mathrm{U}$ and $\mathrm{V}$ be maximal $\boldsymbol{\beta}$-open subsets of a Topological space $\mathrm{X}$, then $U \mathrm{UV}=X$ or $\mathrm{U}=\mathrm{V}$.

\section{Proof:}

If $U U V=X$ then the proof is complete.

If not, i.e. $U U V \neq X$ so we have to show that $\mathrm{U}=\mathrm{V}$.

Since $U U V \neq X$ so $U \subset U U V$ and $V \subset U U V$.

But $\mathrm{U}$ is maximal $\boldsymbol{\beta}$-open set, so $U \mathrm{U} V=X$ or $U U V=U$

Thus $U \mathbf{U}=U$ and so $\mathbf{V} \subset \mathbf{U}$.

Now since $V \subset U U V$ and $\mathrm{V}$ is maximal f-open set, so $U U V=X$ or $U U V=V$,but $U \mathbf{U} \neq X$ so $U \mathrm{U}=V$ and hence $\mathbf{U} \subset \mathbf{V}$

Therefore $\mathrm{U}=\mathrm{V}$.

\section{Theorem (17):}

Let $\mathrm{U}$ be a maximal $\boldsymbol{\beta}$-open and $\mathrm{V}$ be a $\boldsymbol{\beta}$-open subsets of a Topological space $\mathrm{X}$ then $U \mathrm{U} V=X$ or $\mathbf{V} \subset \mathbf{U}$.

\section{Proof:}

If $U U V=X$ then the proof is complete. If $U U V \neq X$ so $U \subset U U V$ and $V \subset U U V$. Since $U$ is maximal $\beta$-open and $U \subset U U V$ so by definition of Maximal $\boldsymbol{\beta}$-open we have that $U U V=X$ or $U U V=U$ but $U U F X X$ so $U \mathrm{UV}=U$ and hence $\mathbf{V} \subset \mathbf{U}$.

Theorem (18):

Let $U$ be a maximal $\boldsymbol{\beta}$-open subset of a Topological space $X$ with $\mathbf{x} \in \mathbf{X} / \mathbf{U}$ then $\mathbf{X} \mathbf{U} \subset \mathbf{V}$ for any $\boldsymbol{\beta}$-open subset of $\mathrm{X}$ with $\mathbf{x} \in \mathbf{V}$.

Proof:

Let $\quad \mathbf{x} \in \mathbf{X} / \mathbf{U}$ and $\quad \mathbf{x} \in \mathbf{V}, \quad$ so $\mathbf{V} \not \subset \mathbf{U}$, thus by (17) we have that $U \mathrm{U} V=X \Rightarrow(X \backslash U) \mathrm{I}(X \backslash V)=\phi$

$\Rightarrow \mathrm{X} \backslash \mathrm{U} \subset \mathrm{V}$. 
Journal of Al-Nahrain University

Science

Theorem (19):

Let $\mathrm{F}$ be a minimal $\boldsymbol{\beta}$-closed and $\mathrm{K}$ be a

$\boldsymbol{\beta}$-closed subsets of a topological space $\mathrm{X}$ then

$F \mathbf{I} K=\phi$ or $\mathbf{F} \subset \mathbf{K}$.

\section{Proof:}

If $F I K=\phi$ then the proof is complete.

If $F \mathrm{I} K \neq \phi$ then we have to show that $\mathbf{F} \subset \mathbf{K}$.

Since $F \mathrm{I} K \neq \phi$ then $F \mathrm{I} K \subset F$ and $F I K \subset K$.

But $\mathrm{F}$ is minimal $\boldsymbol{\beta}$-closed, so we have $F \mathrm{I} K=F$ or $F \mathrm{I} K=\phi$.

Thus $F \mathrm{I} K=F$

\section{so $\mathbf{F} \subset \mathbf{K}$.}

\section{Theorem (20):}

Let $\mathrm{F}$ and $\mathrm{K}$ be minimal $\boldsymbol{\beta}$-closed subsets of a topological space $\mathrm{X}$ then $F \mathrm{I} K=\phi$ or $\mathbf{F}=\mathbf{K}$

\section{Proof:}

If $F I K=\phi$ then the proof is complete.

If $F \mathrm{I} K \neq \phi$ then we have to show that $\mathbf{F}=\mathbf{K}$.

Since $F \mathrm{I} K \neq \phi \quad$ so $\quad F \mathrm{I} K \subset F \quad$ or $F \mathrm{I} K \subset K$.

Since $\mathrm{F}$ is minimal $\boldsymbol{\beta}$-closed so we have $F \mathrm{I} K=F$ or $F \mathrm{I} K=\phi$. But $F \mathrm{I} K \neq \phi$ hence $F \mathrm{I} K=F$ which means $\mathbf{F} \subset \mathbf{K}$.

Now since $\mathrm{K}$ is minimal $\boldsymbol{\beta}$-closed so we have $F \mathrm{I} K=K$ or $F \mathrm{I} K=\phi$. But $F \mathrm{I} K \neq \phi$ hence $F \mathrm{I} K=K$ which means $\mathbf{K} \subset \mathbf{F}$. Therefore $\mathrm{F}=\mathrm{K}$.

\section{Theorem (21):}

Let $\mathrm{U}, \mathrm{V}$ and $\mathrm{W}$ be maximal $\boldsymbol{\beta}$-open subsets of a Topological space $X$ such that $\mathbf{U} \neq \mathbf{V}$, if $U \mathrm{I} V \subset W$, then either $\mathrm{U}=\mathrm{W}$ or $\mathrm{V}=\mathrm{W}$.

\section{Proof:}

Suppose that $U \mathrm{I} V \subset W$, if $\mathrm{U}=\mathrm{W}$ then the proof is complete.

If $\mathbf{U} \neq \mathbf{W}_{\text {we have to show that } \mathrm{V}=\mathrm{W}}$
$V \mathrm{I} W=V \mathrm{I}(X \mathrm{I} W) \quad$ Set Theory

$=V \mathrm{I}[W \mathrm{I}(U \mathrm{UV})]$ by (29)

$=V \mathrm{I}[(W \mathrm{I} U) \mathrm{U}(W \mathrm{I} V)]$ Set Theory

$=(V I$ I $W I U) \mathrm{U}(V I W I V)$ Set Theory

$=(U \mathrm{I} V) \mathrm{U}(V \mathrm{I} W)$ since $U \mathrm{I} V \subset W$

$=(U \mathrm{UW}) \mathrm{I} V$ Set Theory

$=X \mathrm{I} V$ since $U \mathrm{U} W=X$ Thus $\quad V \mathrm{I} W=V$ $=V$

implies $\mathbf{V} \subset \mathbf{W}$ but $\mathrm{V}$ is maximal $\boldsymbol{\beta}$-open therefore $\mathrm{V}=\mathrm{W}$ or $V \mathrm{UW}=X$ but $V \mathrm{U} W \neq X$ so $\mathrm{V}=\mathrm{W}$.

Theorem (22):

$\mathrm{U}, \mathrm{V}$ and $\mathrm{W}$ be maximal $\boldsymbol{\beta}$-open subsets of a Topological space $X$ which are different from each other, then $U \mathrm{I} V \not \subset U I W$

Proof:

Let $U I V \subset U I W$

$\Rightarrow(U \mathrm{I} V) \mathrm{U}(W \mathrm{I} V) \subset(U \mathrm{I} W) \mathrm{U}(W \mathrm{I} V)$

$\Rightarrow(U \mathrm{I} W) \mathrm{U} V \subset(U \mathrm{I} V) \mathrm{U} W$

$\Rightarrow X \mathrm{U} V \subset X \mathrm{UW}$

$\Rightarrow V \subset W$

But $\mathrm{V}$ is maximal $\boldsymbol{\beta}$-open and $\mathrm{W}$ is a proper subset of $\mathrm{X}$ so $\mathrm{V}=\mathrm{U}$, this result contradicts the fact that $\mathrm{U}, \mathrm{V}$ and $\mathrm{W}$ are different from each other. Hence $\mathbf{U}\lceil|\mathbf{V} \not \subset \mathbf{U}| \mid \mathbf{W}$

\section{Theorem (23):}

Let $\mathrm{F}$ be a minimal $\boldsymbol{\beta}$-closed subset of a Topological space $X$, if $\mathbf{X} \in \mathbf{F}$ then $\mathbf{F} \subset \mathbf{K}$ for any $\boldsymbol{\beta}$-closed subset $\mathrm{K}$ of $\mathrm{X}$ containing $\mathrm{X}$.

\section{Proof:}

Suppose $\mathbf{x} \in \mathbf{K}$ and $\mathbf{F} \not \subset \mathbf{K}$ so $F \mathrm{I} K \subset F$ and $F \mathrm{I} K \neq \phi$ since $x \in F \mathrm{I} K$

But $\mathrm{F}$ is minimal $\beta$-closed so $F \mathrm{I} K=F$. or $F \mathrm{I} K=\phi$.

hence $F \mathrm{I} K=F$ which contract the relation $F \mathrm{I} K \subset F$. Therefore $\mathbf{F} \subset \mathbf{K}$.

\section{Theorem:}

Let $\mathrm{F}$ and $\mathbf{F}_{\alpha}(\boldsymbol{\alpha \in A})$ be minimal $\boldsymbol{\beta}$-closed sets if $F \subset \bigcup_{\alpha \in A} F_{\alpha}$ then there exists $\boldsymbol{\alpha}_{\mathbf{0}} \in \mathbf{A}$ such that $\mathbf{F}=\mathbf{F}_{\alpha_{0}}$. 


\section{Proof:}

First we have to show that $F \mathrm{I} F_{\alpha_{0}} \neq \phi$, suppose that $F \mathrm{I} F_{\alpha_{0}}=\phi$ then $\mathbf{F}_{\alpha_{0}} \subset \mathbf{X} \backslash \mathbf{F}$ and so $\quad F \subset \bigcup_{\alpha \in A} F_{\alpha} \subset X \backslash F \quad$ which is a contradiction. So $F \mathrm{I} F_{\alpha_{0}} \neq \phi$. and hence $F \mathrm{I} F_{\alpha_{0}} \subset F$ and $F \mathrm{I} F_{\alpha_{0}} \subset F_{\alpha_{0}}$

since $F \mathrm{I} F_{\alpha_{0}} \subset F$ and $\mathrm{F}$ is minimal $\boldsymbol{\beta}$-closed then $F \mathrm{I} F_{\alpha_{0}}=F$ or $F \mathrm{I} F_{\alpha_{0}}=\phi$

thus $F \mathrm{I} F_{\alpha_{0}}=F$ and hence $\mathbf{F}_{\alpha_{0}} \subset \mathbf{F}$. Now since $\quad F \mathrm{I} F_{\alpha_{0}} \subset F_{\alpha_{0}}$ and $\quad \mathbf{F}_{\alpha_{0}}$ is minimal $\boldsymbol{\beta}$-closed then $F \mathrm{I} F_{\alpha_{0}}=F_{\alpha_{0}}$ or $F \mathrm{I} F_{\alpha_{0}}=\phi$. Thus $F \mathrm{I} F_{\alpha_{0}}=F_{\alpha_{0}}$ and hence $\mathbf{F} \subset \mathbf{F}_{\alpha_{0}}$. Therefore $\mathbf{F}=\mathbf{F}_{\alpha_{0}}$.

\section{$\mathbf{T}_{\boldsymbol{\beta} \text { mir and }} \mathbf{T}_{\boldsymbol{\beta} \text { mar space }}$}

\section{Definition (24):}

A topological space $\mathrm{X}$ is said to be $\mathbf{T}_{\boldsymbol{\beta} \text { mirspace }}$ if every nonempty proper $\boldsymbol{\beta}$-open subset of $\mathrm{X}$ is minimal $\boldsymbol{\beta}$-open set.

\section{Definition (25):}

A topological space $X$ is said to be $\mathbf{T}_{\boldsymbol{\beta}}$ mar space if every nonempty proper $\boldsymbol{\beta}$-open subset of $\mathrm{X}$ is maximal $\boldsymbol{\beta}$-open set.

\section{Example (26):}

Let $X=\{a, b, c\}$ and $\tau=\{\phi,\{\mathbf{a}, \mathbf{b}\},\{\mathbf{c}\}, X\}$ thus $\beta O(X)=\tau$, it is clear that $\{a, b\}$ and $\{c\}$ are maximal and minimal $\boldsymbol{\beta}$-open sets thus the space $X$ is both $\mathbf{T}_{\beta \min }$ and $\mathbf{T}_{\beta \max }$.

\section{Remark (27):}

$\mathbf{T}_{\boldsymbol{\beta} \mathbf{m i n}}$ and $\mathbf{T}_{\boldsymbol{\beta} \mathbf{m a y}}$ spaces are identical.

\section{Theorem (28):}

A space $\mathrm{X}$ is $\mathbf{T}_{\boldsymbol{\beta} \text { mir }}$ if and only if it is T

\section{Proof:}

$\Rightarrow$ Let $X$ is $\mathbf{T}_{\boldsymbol{\beta} \text { mir space. Suppose that } X \text { is }}$ not $\mathbf{T}_{\boldsymbol{\beta} \mathbf{m a}}$, so there is a proper $\boldsymbol{\beta}$-open subset $\mathrm{K}$ of $\mathrm{X}$ which is not maximal, this mean there exist a $\boldsymbol{\beta}$-open subset of $X$ with $\mathbf{K} \subset \mathbf{H} \neq \boldsymbol{\phi}$. Thus we get that $\mathrm{H}$ is not minimal which is contradict of being $X$ is $\mathbf{T}_{\boldsymbol{\beta} \mathbf{m a r}}$.
$\Leftarrow$ Let $X$ is $\mathbf{T}_{\boldsymbol{\beta} \text { may space. Suppose that } X \text { is }}$ not $\mathbf{T}_{\boldsymbol{\beta} \mathbf{m i r}}$, so there is a proper $\boldsymbol{\beta}$-open subset $\mathrm{K}$ of $\mathrm{X}$ which is not minimal, this mean there exist an $\boldsymbol{\beta}$-open subset of $X$ with $\phi \neq \mathbf{H} \subset \mathbf{K}$. Thus we get that $\mathrm{H}$ is not maximal which is contradict of being $X$ is $\mathbf{T}_{\boldsymbol{\beta}}$ mar.

Theorem (29):

A topological space $X$ is $\mathbf{T}_{\boldsymbol{\beta} \text { mir space if }}$ and only if every nonempty proper $\boldsymbol{\beta}$-closed subset of $\mathrm{X}$ is maximal $\boldsymbol{\beta}$-closed set in $\mathrm{X}$.

Proof:

$\Longrightarrow$ let $\mathrm{F}$ be a proper $\boldsymbol{\beta}$-closed subset of $\mathrm{X}$ and suppose $\mathrm{F}$ is not maximal.

So there exists an $\boldsymbol{\beta}$-closed subset $\mathrm{K}$ of $\mathrm{X}$ with $\mathbf{K} \neq \mathbf{X}$ such that $\mathbf{F} \subset \mathbf{K}$.

Thus $\mathbf{X}-\mathbf{K} \subset \mathbf{X}-\mathbf{F}$. Hence $\mathrm{X}-\mathrm{F}$ is a proper $\boldsymbol{\beta}$-open which is not minimal and this contradicts of being $X$ is $\mathbf{T}_{\boldsymbol{\beta} \mathbf{m i n}}$ space.

$\Leftarrow$ Suppose $\mathrm{U}$ is a proper $\boldsymbol{\beta}$-open subset of $\mathrm{X}$. thus $X-U$ is a proper $\boldsymbol{\beta}$-closed subset of $X$, so $\mathrm{X}-\mathrm{U}$ is maximal $\boldsymbol{\beta}$-closed subset of $\mathrm{X}$. and by (15) $U$ is minimal $\boldsymbol{\beta}$-open. thus $X$ is $\mathbf{T}_{\boldsymbol{\beta} \text { min }}$ space.

Theorem (30):

A topological space $X$ is $\mathbf{T}_{\boldsymbol{\beta} \text { maz space if }}$ and only if every nonempty proper $\boldsymbol{\beta}$-closed subset of $\mathrm{X}$ is minimal $\boldsymbol{\beta}$-closed set in $\mathrm{X}$.

\section{Proof:}

$\Longrightarrow$ let $\mathrm{F}$ be a proper $\beta$-closed subset of $\mathrm{X}$, suppose $F$ is not minimal $\boldsymbol{\beta}$-closed in $X$, so there is a proper $\boldsymbol{\beta}$-closed subset of $\mathrm{X}$ such that $\mathbf{K} \subset \mathbf{F}$

Thus $\mathbf{X}-\mathbf{F} \subset \mathbf{X}-\mathbf{K}$ but $\mathrm{X}-\mathrm{K}$ is proper $\boldsymbol{\beta}$-open in $X$ so $X-F$ is not maximal in $X$. Contradiction to the fact $\mathrm{X}-\mathrm{F}$ is maximal $\beta$-open.

$\Leftarrow$ let $\mathrm{U}$ be a proper $\boldsymbol{\beta}$-open subset of $\mathrm{X}$, then $\mathrm{X}$-U is a proper $\boldsymbol{\beta}$-closed subset of $\mathrm{X}$ and so it is minimal $\boldsymbol{\beta}$-closed set. By (15) we get that $\mathrm{U}$ is maximal $\boldsymbol{\beta}$-open.

\section{Theorem (31):}

Every pair of different minimal $\beta$-open sets of $\mathbf{T}_{\boldsymbol{\beta} \mathbf{m i r}}$ are disjoint. 
Journal of Al-Nahrain University

Science

Proof: Let $\mathrm{U}$ and $\mathrm{V}$ be minimal f-open subsets of $\mathbf{T}_{\boldsymbol{\beta} \text { mirspace }} \mathrm{X}$ such that $\mathbf{U} \neq \mathbf{V}$ to show that $U \mathrm{I} V=\phi$ suppose not i.e. $U \mathrm{I} V \neq \phi$.

So $U I V \subset U$ and $U I V \subset V$.

Since

$U \mathrm{I} V \subset U$ and $\mathrm{U}$ is minimal $\boldsymbol{\beta}$-open then $U \mathrm{I} V=U$ or $U \mathrm{I} V=\phi$ thus $U \mathrm{I} V=U$.

Now since $U \mathrm{I} V \subset V$ and $\mathrm{V}$ is minimal $\beta$-open then $U \mathrm{I} V=V$ or $U \mathrm{I} V=\phi$ thus $U I V=V$.

Hence we get that $\mathrm{U}=\mathrm{V}$ this result contradicts the fact that $\mathrm{U}$ and $\mathrm{V}$ are different. Therefore $U I V=\phi$.

\section{Theorem (32):}

Union of every pair of different maximal

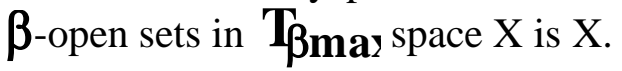

Proof: Let $\mathrm{U}$ and $\mathrm{V}$ be maximal $\boldsymbol{\beta}$-open subsets of $\mathbf{T}_{\boldsymbol{\beta} \text { may space } X \text { such that }} \mathbf{U} \neq \mathbf{V}$ to show that $U U V=X$ suppose not i.e. $U V \neq X$.

So $U \subset U U V$ and $V \subset U U V$.

Since $U \subset U U V$ and $U$ is maximal $\beta$-open then $U U V=U$ or $U U V=X$.

Thus $U U V=U \ldots$ (1).

Now since $V \subset U U V$ and $\mathrm{V}$ is maximal $\beta$-open then $U U V=V$ or $U \mathrm{I} V=X$

Thus $U U V=V$... (2)

Hence from (1) and (2) we get that $\mathrm{U}=\mathrm{V}$ this result contradicts the fact that $\mathrm{U}$ and $\mathrm{V}$ are different. Therefore $U I V=X$.

Continuity with Minimal and Maximal $\boldsymbol{\beta}$-open Sets

Definition (33):

Let $\mathrm{X}$ and $\mathrm{Y}$ be topological spaces, a map $\mathbf{f}: \mathbf{X} \rightarrow \mathbf{Y}$ is called minimal $\boldsymbol{\beta}$-continuous if $\mathbf{f}^{-1}(\mathbf{U})$ is minimal $\boldsymbol{\beta}$-open in $\mathrm{X}$ for any open subset U of Y.

\section{Example (34):}

Let $\mathrm{X}=\mathrm{Y}=\{\mathrm{a}, \mathrm{b}, \mathrm{c}\}$ and $\mathbf{f}:(\mathbf{X}, \tau) \rightarrow(\mathbf{Y}, \boldsymbol{\sigma})$ is the identity map, where $\tau=\{\phi,\{a\},\{a, b\}, X\}$ and $\boldsymbol{\sigma}=\{\phi,\{a\}, Y\}$ then $\mathrm{f}$ is minimal $\boldsymbol{\beta}$-continuous since the only proper open subset of $\mathrm{Y}$ is $\{\mathrm{a}\}$ and $\left.\mathbf{f}^{-1}(\mathbf{a}\}\right)=\{\mathbf{a}\}$ is minimal $\boldsymbol{\beta}$-open in $X$.
Definition (35):

Let $X$ and $Y$ be topological spaces, a map $\mathbf{f}: \mathbf{X} \rightarrow \mathbf{Y}_{\text {isn }}$ called maximal $\boldsymbol{\beta}$-continuous if $\mathbf{f}^{-1}(\mathbf{U})$ is maximal $\boldsymbol{\beta}$-open in $\mathrm{X}$ for any open subset $\mathrm{U}$ of $\mathrm{Y}$.

\section{Example (36):}

Let $\mathrm{X}=\mathrm{Y}=\{\mathrm{a}, \mathrm{b}, \mathrm{c}\}$ and $\mathbf{f}:(\mathbf{X}, \tau) \rightarrow(\mathbf{Y}, \boldsymbol{\sigma})$ is the identity map, where $\tau=\{\phi,\{a\},\{b\},\{a, b\}, X\}$ and $\boldsymbol{\sigma}=\{\phi,\{a, c\}$, $\mathrm{Y}\}$ then $\mathrm{f}$ is maximal $\boldsymbol{\beta}$-continuous since the only proper open subset of $\mathrm{Y}$ is $\{\mathrm{a}, \mathrm{c}\}$ and $\mathbf{f}^{-1}(\{\mathbf{a}, \mathbf{c}\})=\{\mathbf{a}, \mathbf{c}\}$ is maximal $\boldsymbol{\beta}$-open in $\mathrm{X}$.

Theorem (37):

Every minimal $\boldsymbol{\beta}$-continuous map is $\boldsymbol{\beta}$-continuous.

\section{Proof:}

Let $\mathbf{f}: \mathbf{X} \rightarrow \mathbf{Y}$ be a minimal $\boldsymbol{\beta}$-continuous map and $U$ be open subset of $Y$. then $\mathbf{f}^{-1}(\mathbf{U})$ is minimal $\boldsymbol{\beta}$-open in $X$ and so $\mathbf{f}^{-1}(\mathbf{U})$ is $\boldsymbol{\beta}$-open subset of $\mathrm{X}$

\section{Remark 38:}

The converse is not true in general as in the following example.

\section{Example (39):}

Let $\mathrm{X}=\mathrm{Y}=\{\mathrm{a}, \mathrm{b}, \mathrm{c}\}$ and $\mathbf{f}:(\mathbf{X}, \tau) \rightarrow(\mathbf{Y}, \boldsymbol{\sigma})$ is the identity map, where $\tau=\{\phi,\{a\},\{c\},\{a, c\}, X\}$ and $\boldsymbol{\sigma}=\{\phi,\{a, c\}$, $\mathrm{Y}$ \} then $\mathrm{f}$ is $\boldsymbol{\beta}$-continuous but $\mathrm{f}$ is not minimal $\boldsymbol{\beta}$-continuous since $\left.\mathbf{f}^{-1}(\boldsymbol{a}, \mathbf{c}\}\right)=\{\mathbf{a}, \mathbf{c}\}$ is not minimal $\boldsymbol{\beta}$-open since $\{\mathbf{a}\} \in \boldsymbol{\beta} \mathbf{O}(\mathbf{X})$ and $\phi \neq\{\mathbf{a}\} \subset\{\mathbf{a}, \mathbf{c}\}$.

\section{Theorem (40):}

Let $\mathrm{X}$ and $\mathrm{Y}$ be topological spaces, if $\mathbf{f}: \mathbf{X} \rightarrow \mathbf{Y}$ is an $\boldsymbol{\beta}$-continuous onto map and $X$ is $T_{\beta}$ min space then $f$ is minimal $\boldsymbol{\beta}$-continuous.

\section{Proof:}

It is clear that the inverse image of $\phi$ and $Y$ are f-open subsets of $\mathrm{X}$. So let $\mathrm{U}$ be a proper open subset of Y. Since $\mathrm{f}$ is f-continuous so 
$\mathbf{f}^{-1}(\mathbf{U})$ is proper f-open subset of $X$, but $X$ is $\mathbf{T}_{\boldsymbol{\beta} \mathbf{m i r}}$ so $^{-1}(\mathbf{U})$ minimal f-open.t.

Remark (41):

The converse is not true in general as in the following example.

\section{Example (42):}

In (34) $\mathrm{f}$ is minimal $\mathrm{f}$-continuous but $\mathrm{X}$ is not $\mathbf{T}_{\beta}$ mir.

\section{Theorem (43):}

Let $\mathrm{X}$ and $\mathrm{Y}$ be topological spaces, if $\mathbf{f}: \mathbf{X} \rightarrow \mathbf{Y}$ is a $\boldsymbol{\beta}$-continuous onto map and $\mathrm{X}$

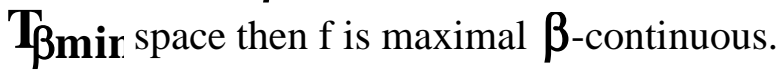

\section{Proof:}

It is clear that the inverse image of $\phi$ and $Y$ are $\boldsymbol{\beta}$-open subsets of $X$. So let $U$ be a proper open subset of $Y$. Since $f$ is $\boldsymbol{\beta}$-continuous so $\mathbf{f}^{-1}(\mathbf{U})$ is a proper $\boldsymbol{\beta}$-open subset of $X$ but $X$ is $T_{\beta \min }$ so $\mathbf{f}^{-1}(\mathbf{U})$ is maximal $\boldsymbol{\beta}$-open.

\section{Remark (44):}

The converse is not true in general as in the following example.

Example (45): In (36) $\mathrm{f}$ is maximal $\boldsymbol{\beta}$-continuous but $X$ is not $\mathbf{T}_{\boldsymbol{\beta} \text { min space. }}$

\section{Theorem (46):}

Every maximal $\boldsymbol{\beta}$-continuous map is $\boldsymbol{\beta}$-continuous.

\section{Proof:}

Let $\mathbf{f}: \mathbf{X} \rightarrow$ Ybe a maximal $\boldsymbol{\beta}$-continuous map and $U$ be open subset of $Y$. then $\mathbf{f}^{-1}(\mathbf{U})$ is maximal $\boldsymbol{\beta}$-open in $X$ and so $\mathbf{f}^{-1}(\mathbf{U})$ is $\boldsymbol{\beta}$-open subset of $\mathrm{X}$.

\section{Remark (47):}

The Converse is not true in general as in the following example.

\section{Example (48):}

Let $\mathrm{X}=\mathrm{Y}=\{\mathrm{a}, \mathrm{b}, \mathrm{c}\}$ and $\mathbf{f}:(\mathbf{X}, \tau) \rightarrow(\mathbf{Y}, \sigma)$ is the identity map, then where $\tau=\{\phi,\{a\},\{a, c\}, X\}$ and $\sigma=\{\phi,\{a\}$, $\mathrm{Y}\}$ then $\mathrm{f}$ is $\boldsymbol{\beta}$-continuous but $\mathrm{f}$ is not maximal $\boldsymbol{\beta}$-continuous since $\left.\mathbf{f}^{-1}(\mathbf{a}\}\right)=\{\mathbf{a}\}$ is not maximal $\boldsymbol{\beta}$-open since $\phi \neq\{\mathbf{a}, \mathbf{c}\} \supset\{\mathbf{a}\}$.

\section{Remark (49):}

Minimal $\boldsymbol{\beta}$-continuous and maximal $\boldsymbol{\beta}$-continuous maps are independent of each other and the following examples show that.

\section{Example (50):}

In (36) $\mathrm{f}$ is maximal $\boldsymbol{\beta}$-continuous since $\left.\mathbf{f}^{-1}(\boldsymbol{a}, \mathbf{c}\}\right)=\{\mathbf{a}, \mathbf{c}\}$ is $\boldsymbol{\beta}$-open but $\mathrm{f}$ is not minimal $\boldsymbol{\beta}$-continuous.

\section{Example (51):}

In (34) $\mathrm{f}$ is minimal $\boldsymbol{\beta}$-continuous but it is not maximal $\boldsymbol{\beta}$-continuous

since $\mathbf{f}^{-1}(\{\mathbf{b}\})=\{\mathbf{b}\}$ is not maximal $\boldsymbol{\beta}$-open in $\mathrm{X}$.

\section{Theorem (52):}

Let $\mathbf{f}: \mathbf{X} \rightarrow \mathbf{Y}$ be a map and $\mathrm{X}$ and $\mathrm{Y}$ be topological spaces, then $\mathrm{f}$ is maximal (resp. minimal) $\boldsymbol{\beta}$-continuous if and only if $\mathbf{f}^{-1}(\mathbf{F})$ is minimal (resp. maximal) $\boldsymbol{\beta}$-closed subset of $\mathrm{X}$ for each closed subset $\mathrm{F}$ of $\mathrm{Y}$.

Proof: $\Rightarrow$ let $\mathrm{F}$ be a closed set in $\mathrm{Y}$. thus $\mathrm{Y}-\mathrm{F}$ is open and so $\mathbf{f}^{-1}(\mathbf{Y}-\mathbf{F}$ ) is maximal (resp. maximal) $\boldsymbol{\beta}_{\text {-open. but }} \mathbf{f}^{-1}(\mathbf{Y}-\mathbf{F})=\mathbf{X}-\mathbf{f}^{-1}(\mathbf{F})$ so $\mathbf{f}^{-1}(\mathbf{F})$ is minimal (resp. maximal) $\boldsymbol{\beta}$ closed.

\section{Theorem (53):}

Let $\mathrm{X}, \mathrm{Y}$ and $\mathrm{Z}$ be topological spaces, if $\mathbf{f}: \mathbf{X} \rightarrow \mathbf{Y}_{\text {is a minimal ( respect. maximal) }}$ $\boldsymbol{\beta}$-continuous map and $\mathbf{g :} \mathbf{Y} \rightarrow \mathbf{Z}$ is a continuous map then $\mathbf{g} \circ \mathbf{f}: \mathbf{X} \rightarrow \mathbf{Z}$ is a minimal (resp. maximal) $\boldsymbol{\beta}$-continuous map. Proof: Let $\mathrm{U}$ be an open subset of $\mathrm{Z}$, since $\mathrm{g}$ is continuous so $\mathbf{g}^{-1}(\mathbf{U})$ is an open subset of $Y$. But $\mathrm{f}$ is minimal (respect. maximal) $\boldsymbol{\beta}$-continuous thus $\mathbf{f}^{-1}\left(\mathbf{g}^{-1}(\mathbf{U})\right)=(\mathbf{g} \circ \mathbf{f})^{-1}$ is a minimal (respect. maximal) $\boldsymbol{\beta}$-open subset of $\mathrm{X}$.

\section{Conclusion}

In this paper we get some theorems presented to reveal many various properties of 


\section{Science}

the minimal $\boldsymbol{\beta}$-open and maximal $\boldsymbol{\beta}$-open and their complements and we defined two types of topological spaces and finally we defined continuity over the new sets which produced here.

\section{References}

[1] Nakaoka F., and Oda N., "Some applications of minimal open sets", Int. J. Math. Math. Sci. 27-8, 471-476, 2001.

[2] Nakaoka F., and Oda N., “ Some properties of maximal open sets", Int. J.Math. Math. Sci. 21, 1331-1340, 2003.

[3] Nakaoka F., and Oda N., "On minimal closed sets", Proceeding of Topological Spaces and its Applications, 5, 19-21, 2003.

[4] Munkers J.R., "Topology", Pearson Education (India), 2001.

[5] Abd El-Monsef M. E., El-Deeb S. N., Mahmoud R. A., " $\boldsymbol{\beta}$-open sets and $\boldsymbol{\beta}$-continuous mappings", Bulletin of the Faculty of Science, Assiut University, 12, pp.77-90, 1983.

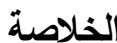

يناقش البحث الحالي أنواعا جديدة من المجموعات

ويتعامل معها. وكل هذه المفاهيم تعتمد على مفهوم

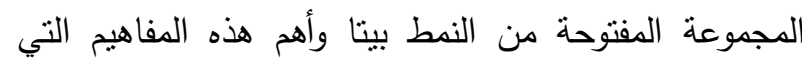

قدمت في هذا البحث هي المجموعة المفتوحة بيتا الاصغرية

و المجموعة المفتوحة بيتا الأكبرية. كما تم تقديم نوعين

جديدين من الفضاءات التبولوجيا سميت الفضاء من النمط بلها

T T $\mathbf{T}_{\beta \max }$

نمطين جديدين من الدوال المستمرة هما الدالة المستمرة

الاصغرية من النمط بيتا و الدالة المستمرة الاكبرية من النمط هن لهن

بينا، بالاضافة الى ذلك تم دراسة بعض الخواص الاساسية

للمفاهيم المطروحة في هذا البحث. 